\title{
The Standardization of the Vestibular Evoked Myogenic Potential
}

DOI: $10.3766 /$ jaaa.30.5.1

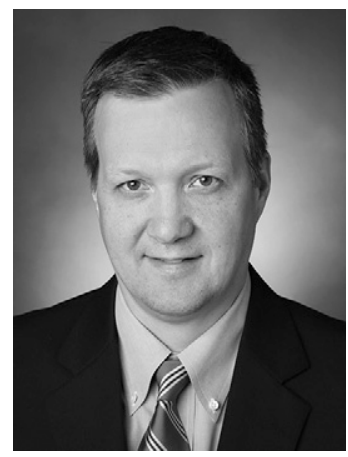

$\mathrm{I}$

$\mathrm{n}$ the balance laboratories of most tertiary care clinics and now more commonly in private practice environments, vestibular evoked myogenic potentials (VEMPs) have become a standard assessment in the vestibular test battery. This is due to the fact that evokedpotential (EP) systems are already being used to screen for hearing impairment and estimate audiometric thresholds. At the time of this writing, most EP system manufacturers have developed VEMP software and/or hardware add-ons that can be purchased outright or obtained using a research IRB. Industry has worked to develop specialized modules for VEMP waveform analysis and electromyography correction, however, as of yet there is little consistency with which the VEMP response is being collected and interpreted by clinicians.

There are a number of reasons for this development. One that may be largely responsible is the continual stream of new research reports detailing the discovery of more optimal stimulating or recording parameters of the response. When students learn about VEMPs in the classroom, they are often assigned a textbook with a single chapter on the topic. However, while textbook chapters are written broadly with the most contemporary knowledge at the time, it can take a year or even two to bring a textbook to publication. This creates a situation where students may be learning about VEMP techniques that are no longer considered appropriate or even used clinically. One alternative to reading book chapters is to stalk PubMed and set up reminders that will send the clinician every article from a journal that has the word VEMP in the title or abstract. While this seems like a logical and efficient way to stay current on the most recent VEMP research, it can become overwhelming. I know because I currently have an e-mail inbox filled with recently published VEMP studies that were automatically sent to me and now need to be read. When reading studies in peer-reviewed articles, the clinician must also learn to be a smart consumer of research. That is, each report should be read critically to determine its merits and deficiencies and whether or not the findings warrant being incorporated into that individual's clinic.
Fortunately there are groups that have worked to provide the balance community with guidelines that are evidence-based summaries that deliver clearly and concisely summarize the most current techniques to optimize VEMP recording and analysis. One of the first formal guidelines for VEMPs was published by Papathanasiou et al (2014) entitled the "International guidelines for the clinical application of cervical vestibular evoked myogenic potentials: an expert consensus report." This was an excellent overview of the knowledge at that time. Another example is a recent thorough review by Rosengren et al (2019) published this year. This article presents the most current recommendations regarding the recording and stimulating parameters of VEMPs, as well an overview of how VEMPs can be used to assist in the diagnosis of vestibular disorders. The article also has a section covering common pitfalls when performing the test. This is a must read for anyone who records VEMPs in the clinic.

In this regard, Romero and colleagues report their findings on the effect of stimulus polarity on the ocular VEMP (oVEMP) response. The oVEMP can be evoked using air or bone conducted stimuli and is recorded from an extraocular eye muscle (i.e., the contralateral inferior oblique). While there have been numerous studies detailing the effects of the electrode montage on oVEMP response metrics, there are far fewer reports describing the effect of stimulus polarity on amplitude and latency. Sandhu et al (2013) first reported a novel electrode montage that has been shown to generate superior responses compared to earlier reported montages. The investigators showed that by placing the non-inverting electrodes near the belly of the inferior oblique muscle and the inverting over the tendon of the muscle (i.e., inner canthus) the amplitude of the oVEMP response could be significantly enhanced. This method for recording the oVEMP is gaining popularity; however, until now the effect of stimulus polarity on this novel montage was unknown. We encourage you to read the article in this issue entitled "Impact of Stimulus Polarity on oVEMP Response using the 
Belly-Tendon Electrode Montage.” The findings in this report should be considered for those of us that currently have oVEMPs included in our practice. Studies such as this are critical for continually improving our ability to record these responses. In the future there is a high likelihood that there will be a VEMP code for billing in the US. When that time arrives, there will undoubtedly be a significant increase in the number of clinics offering these tests. It is therefore important that we continue to investigate these responses and report the results in peer-reviewed articles as Romero and colleagues have in this issue. Just as important will be the need for well-respected groups like those mentioned earlier to provide us with review papers synthesizing new research and to provide updates to current guidelines.

Devin L. McCaslin, Ph.D. Deputy Editor-in-Chief

\section{REFERENCES}

Papathanasiou ES, Murofushi T, Akin FW, Colebatch JG. (2014) International guidelines for the clinical application of cervical vestibular evoked myogenic potentials: an expert consensus report. Clin Neurophysiol 125(4):658-666.

Rosengren SM, Colbatch JG, Young AS, Govender S, Welgampola MS. (2019) Vestibular evoked myogenic potentials in practice: Methods, pitfalls and clinical applications. Clin Neurophysiol Pract 4:47-68. 